Original Article

Artigo Original

\author{
Ahmed Nagy ${ }^{1,2}$ \\ Melanie Peladeau-Pigeon ${ }^{1}$ \\ Teresa Josephine Valenzano ${ }^{1,3}$ \\ Ashwini Marini Namasivayam ${ }^{1,3}$ \\ Catriona Margaret Steele $e^{1,3,4}$
}

Keywords

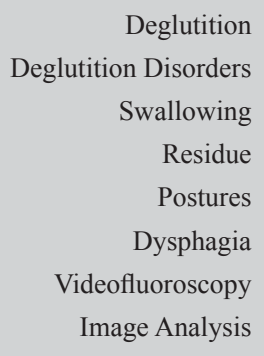

Correspondence address:

Catriona M. Steele

550 University Avenue, \#12-101,

Toronto, ON, Canada, M5G 2A2.

E-mail: catriona.steele@uhn.ca

Received: December 04, 2015

Accepted: December 13, 2015

\section{The effectiveness of the head-turn-plus- chin-down maneuver for eliminating vallecular residue}

\begin{abstract}
Purpose: When swallowing efficiency is impaired, residue accumulates in the pharynx. Cued or spontaneous swallows in the head neutral position do not always successfully clear residue. We investigated the impact of a novel maneuver on residue clearance by combining a head turn with the chin down posture. Methods: Data were collected from 26 participants who demonstrated persistent vallecular residue after an initial head neutral clearance swallow in videofluoroscopy. Participants were cued to perform a head-turn-plus-chin-down swallow, with the direction of head turn randomized. Pixel-based measures of residue in the vallecular space before and after the maneuver were made on still frame lateral images using ImageJ software. Measures of $\%$ full and the Normalized Residue Ratio Scale (NRRS) were extracted. Univariate analyses of variance were used to detect significant reductions in residue. Results: On average, pre-maneuver measures showed residue filling 56-73\% of the valleculae, depending on stimulus consistency (NRRS scores: $0.2-0.4$ ). More than $80 \%$ of pre-swallow measures displayed NRRS ratios $>0.06$, a threshold previously linked to increased risk of post-swallow aspiration. Conclusion: The head-turn-plus-chin-down maneuver achieved significant reductions in residue for thin and nectar-thick fluids, suggesting that this maneuver can be effective in reducing persistent vallecular residue with these consistencies.
\end{abstract}

\section{INTRODUCTION}

Videofluoroscopy is the current gold-standard method for evaluating swallowing pathophysiology in people with dysphagia, using a standardized protocol. Due to the radiographic exposure associated with this tool, videofluoroscopic evaluations must be restricted in duration, typically involving between 3 and 11 boluses per assessment $t^{(1-3)}$. It is within this very narrow window that treatment decisions have to be made. One of those decisions involves the choice of compensatory techniques that are appropriate to address the specific pathophysiology demonstrated by the patient. Among these, postural modifications are frequently considered as a means of reducing penetration-aspiration risk or improving bolus clearance ${ }^{(4-13)}$.

When swallowing efficiency is impaired, residue collects in the spaces of the pharynx (the valleculae and pyriform sinuses). In a study by Perlman et al. ${ }^{(14)}$, vallecular residue and epiglottic dysfunction were found to have the highest co-occurrence rate with aspiration $(80 \%)$, of all parameters measured. The presence of residue has also been shown to have prognostic value in predicting the outcome of rehabilitation for stroke patients with dysphagia. Park et al. ${ }^{(15)}$ reported that stroke patients with greater residue in the valleculae and pyriform sinuses had poorer treatment outcomes following a rehabilitation protocol involving neuromuscular electrical stimulation.

\footnotetext{
Study carried out at Toronto Rehabilitation Institute, University Health Network - Toronto (ON), Canada. ${ }^{1}$ Toronto Rehabilitation Institute, University Health Network - Toronto (ON), Canada.

${ }^{2}$ University of Fayoum, Fayoum, Egypt.

${ }^{3}$ University of Toronto - Toronto (ON), Canada.

${ }^{4}$ Bloorview Research Institute - Toronto (ON), Canada.
}

Financial support: Provincial Rehabilitation Research Program from the Ministry of Health and Long-term Care in Ontario, Canada.

Conflict of interests: nothing to declare. 
Residue is thought to represent a risk for subsequent post-swallow aspiration $^{(16)}$. In a retrospective analysis of 156 thin-liquid clearing swallows from patients with neurogenic dysphagia, Molfenter and Steele ${ }^{(16)}$ found that Normalized Residue Ratio Scale (NRRS) ${ }^{(17)}$ values $>0.09$ of residue from the previous swallow in the vallecular space were associated with double the risk of penetration-aspiration on the subsequent swallow.

Several studies in swallowing literature have investigated the beneficial effects of various interventional postures on the safety and efficiency of swallowing ${ }^{(18-20)}$. Ohmae et al. ${ }^{(8)}$ studied the effects of head rotation on pharyngeal function in normal swallowing using pharyngeal manometry; they concluded that pharyngeal peak pressure was significantly increased towards the side of the head rotation, while the pharyngeal pressures opposite to the side of rotation were not affected. Head rotation was also reported to produce a significant reduction in upper esophageal sphincter pressure. Ertekin et al. ${ }^{(12)}$ investigated the effect of different head positions on oropharyngeal swallowing using electromyography. Swallowing performance improved significantly in $67 \%$ of the patients with unilateral lower cranial nerve lesions when the head was rotated towards the paretic side. In patients with bilateral symptoms, a significant improvement in swallowing performance occurred in $50 \%$ of patients using a chin down posture.

In a study by Balou et al. ${ }^{(9)}$, the immediate effects of partial and complete head rotation, as well as partial and complete chin tuck were investigated. Swallowing pressure amplitudes and durations were measured in 10 healthy individuals in the pharynx and the upper esophageal sphincter. The results showed that both partial and complete head rotations increased the duration of UES relaxation, which was interpreted to reflect stretch on the cricopharyngeus muscle. Pharyngeal pressures were not affected by head rotation. With the chin down posture, their results showed a slight, but non-significant increase in contraction pressure at a sensor located at the level of the valleculae, together with decreased pressures at the upper esophageal sphincter. Due to the involvement of only healthy participants, the impact of these maneuvers on residue was not explored ${ }^{(9)}$.

In clinical practice, when residue is detected in the valleculae, it is typical for either spontaneous or cued clearing swallows to be attempted in the head neutral posture. The result is either effective clearance or persistent residue. In this study we investigated the utility of a novel maneuver, combining a head-turn with the chin-down posture, for reducing post swallow vallecular residue using a cued clearance swallow. This combined head-turn-pluschin-down posture was first suggested by Logemann in her Manual for the Videofluorographic Study of Swallowing(21, p. 50); however, to our knowledge, the maneuver has not previously been empirically investigated. Our study was prompted by anecdotal clinical observations that this technique might be effective. We were curious to determine whether the benefits reported by Ertekin et al. ${ }^{\left({ }^{12}\right)}$ for each component of the maneuver individually, would combine for greater benefit in patients with post-swallow vallecular residue. Our hypotheses were:

- that the effect of turning the head to the side, and tucking the chin down would aid in squeezing material out of the valleculae on a clearing swallow, thereby reducing the amount of residue;
- that the effectiveness of the maneuver would differ according to the consistency of the bolus.

\section{MATERIALS AND METHODS}

A total of 73 patients consented to be screened for this study during routine clinical videofluoroscopy. Of these, 26 patients had at least one qualifying swallow involving persistent vallecular residue, leading to exploration of the head-turn-plus-chin-down maneuver. Demographics for these 26 qualifying participants were as follows: 23 males, 3 females; mean age: 69 years (range: 21 to 87). Primary diagnoses included stroke $(\mathrm{N}=13)$; acquired brain injury $(\mathrm{N}=7)$, including 5 cases of traumatic brain injury and single cases of encephalopathy and intracranial hemorrhage; inclusion body myositis $(\mathrm{N}=1)$; and 5 elderly patients each diagnosed with decompensation and dysphagia symptoms secondary to acute care hospitalization for sepsis, lung transplant, knee replacement, radiation treatment for tonsillar cancer and radiation treatment for colorectal cancer.

A qualifying swallow was defined as a swallow with persistent vallecular residue, which was operationally defined as the presence of a visible fluid level (i.e., more than a thin line coating the walls of the vallecular space) after a single spontaneous or cued clearing swallow in the head neutral position. Piecemeal swallows, for which additional material was added from the oral cavity on the secondary swallow, were excluded. The presence of pyriform sinus residue was an exclusion criterion given evidence that a chin down posture may squeeze residue from this location into the airway ${ }^{(5)}$. Additional exclusion criteria included a history of cervical spine surgery (which might contraindicate bending or turning of the head), the presence of a tracheostomy tube in situ, current pregnancy, and inability to follow procedural instructions secondary to cognitive impairment. After demonstration of a qualifying swallow, participants were cued to perform a head-turn-pluschin-down clearing swallow with the direction of the head turn assigned in a randomized fashion across participants. Approval from the local institutional research ethics board was obtained before starting the study.

Standardized recipes for preparing barium stimuli were used. The thin liquid barium was a $22 \% \mathrm{w} / \mathrm{v}$ liquid barium suspension prepared using Liquid Polibar Plus ${ }^{\circledR}$ (Bracco Diagnostics Inc., Monroe Township, New Jersey). Nectar, honey, and spoon thick barium were prepared in a $40 \% \mathrm{w} / \mathrm{v}$ barium concentration by mixing Bracco EZ-HD® powder into the corresponding facility kitchen product. Minced solid stimuli were prepared by mixing $125 \mathrm{ml}$ of minced vegetables with $5 \mathrm{ml}$ of undiluted Liquid Polibar Plus, and an additional teaspoon of EZ-HD powder. Bread and cookies were smeared with a $100 \% \mathrm{w} / \mathrm{v}$ barium concentration paste prepared using pudding mixed with EZ-HD powder. The videofluoroscopy was performed at 30 pulses per second and recorded at a resolution of 30 frames per second on the KayPENTAX Digital Swallowing Workstation.

Data processing involved blinded review of each videofluoroscopy by licensed speech-language pathologists previously trained to a high level of inter-rater agreement (i.e., ICCs $>0.8$ ) for pixel-based measures of residue severity on a training set of 
videofluoroscopies that was unrelated to the current data set. The rating process involved the identification of still-image head neutral frames corresponding to the end of each swallow (initial head neutral and subsequent qualifying clearing swallows), and pixel-based measures of residue on those frames using ImageJ software. The residue measures involved tracing both the residue area and the surrounding spatial housing of the valleculae, as well as tracing the length of the $\mathrm{C} 2-\mathrm{C} 4$ cervical spine as an anatomical scalar reference ${ }^{(17)}$, as illustrated in Figure 1. These measures were then entered into the formulae for determining the $\%$-full and NRRS-vallecular parameters.

\section{ANALYSIS}

Descriptive statistics for the parameters of interest (\%-full, NRRSv) were calculated for pre- and post-maneuver measures by consistency. Despite the use of a standard sequence of bolus testing in videofluoroscopy (beginning with thin liquids and proceeding to nectar-thick, honey-thick and solid stimuli as deemed appropriate by the attending clinician), not all consistencies led to residue and qualifying swallows in each individual participant. Consequently, we decided to divide the data into two consistency clusters: a) "liquids" (thin and nectar-thick stimuli), $\mathrm{N}=19$; and b) "semi-solids" (honey-thick liquids and all solid stimuli), $\mathrm{N}=46$. Statistical analyses were performed using IBM SPSS Statistics version 21. Repeated measures analyses of variance with a factor of consistency (liquids; semi-solids) and a repeated factor of time-point (pre-, post-) were used to detect significant reductions in the residue with the maneuver measured using both variables ( $\%$ full, NRRS). Post-hoc examinations explored the frequency with which the maneuver reduced residue to below the NRRS $>0.09$ threshold previously reported by Molfenter and Steele to delineate aspiration risk on the subsequent clearing swallow ${ }^{(16)}$

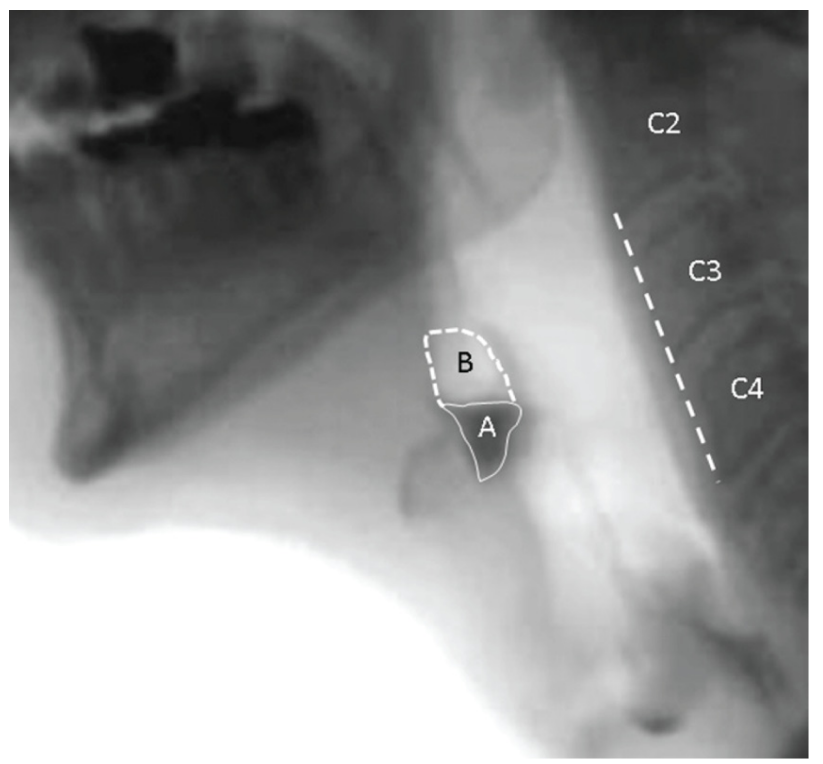

Figure 1. Image showing the components traced in ImageJ for determining vallecular residue measures of \%-full and the Normalized Residue Ratio Scale

\section{RESULTS}

Descriptive statistics for the parameters of interest are found in Table 1.

\section{Percent vallecular fullness}

On average, pre-maneuver measures, at the end of the initial head-neutral clearing swallow, showed residue filling of $62 \%-66 \%$ of the valleculae, depending on stimulus consistency. Post-maneuver measures, at the end of the qualifying swallow, showed a reduction of residue to mean values of $33 \%$ for liquids and $53 \%$ for the semisolid stimuli (Figure 2). A statistically significant main effect of time-point $(\mathrm{F}(1,29)=14.09, \mathrm{p}=0.001)$ and a significant time-point by consistency effect $(\mathrm{F}(1,29)=5.09$, $\mathrm{p}=0.03$ ) were found, illustrating the greater reduction of residue with the liquid consistencies. No main effect of consistency was found.

\section{Normalized residue ratio scale - vallecular (NRRSv)}

Pre-maneuver NRRSv measures averaged 0.34 for the liquid consistencies and 0.30 for the semi-solid consistencies. Post-maneuver measures dropped to 0.098 for the liquid stimuli and 0.26 for the semi-solids (Figure 3). As with the \%-full measures, these differences represented a statistically significant main effect of time-point $(\mathrm{F}(1,29)=11.56, \mathrm{p}=0.002)$ and a significant time-point by consistency effect $(\mathrm{F}(1,29)=5.74$, $p=0.02)$. There was no main effect of consistency.

Cross-tabulation of the NRRS results according to the $<$ vs. $>0.09$ threshold showed that pre-maneuver residue was above this risk threshold in $87.5 \%$ of the liquid cases and $82.6 \%$ of the semi-solid cases. Post-maneuver, residue remained above this risk threshold in $68.5 \%$ of the liquid cases and $71.6 \%$ of the semi-solid cases. If the NRRS $<0.09$ threshold is applied as the definition of effective residue resolution, these data show effective resolution of liquid residue $29 \%$ of the time, and effective resolution of semi-solid residue $11 \%$ of the time.

\section{DISCUSSION}

The study explores the usefulness of a novel intervention targeting the clearance of persistent vallecular residue. Post-swallow residue is a potential source of subjective patient discomfort, and is highlighted by earlier studies as a potential risk for secondary aspiration ${ }^{(16)}$. We based our hypotheses on anecdotal clinical observations, and on scientific literature that separately explores the physiological effects of each component of the head-turn-plus-chin-down maneuver. The results of the study show significant reductions in post-swallow measures of vallecular residue, particularly for thin and nectar-thick liquids, with clinically effective resolution below a previously-reported aspiration-risk threshold in $11-29 \%$ of cases. These findings are clinically important and have potential to influence clinical decision making in videofluoroscopy.

In this study we chose to evaluate the impact of the head-turn-chin-down technique in patients with problematic and persistent vallecular residue. The results confirm that the 
Table 1. Means and $95 \%$ confidence intervals for the \%-full and Normalized Residue Ratio Scale - Vallecular (NRRSv) parameters by consistency and time-point

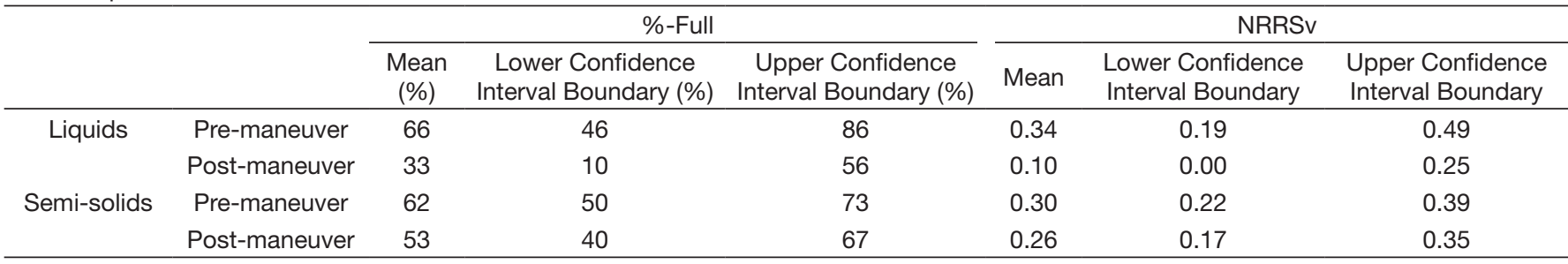

Caption: This still lateral videofluoroscopic image shows the features that were traced using ImageJ to derive measures of vallecular residue severity. The measure of \%-full is calculated by dividing area A by the sum of areas A and B. This ratio is then multiplied by a normalized measure of the residue (area A) using a cervical spine scalar scalar shown by the dashed line running from the anterior inferior corner of $\mathrm{C} 2$ down to the anterior inferior corner of $\mathrm{C} 4$. The formula for the Normalized

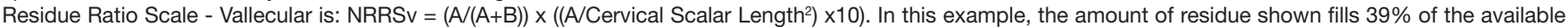
space in the valleculae, and represents an NRRSv measure of 0.15

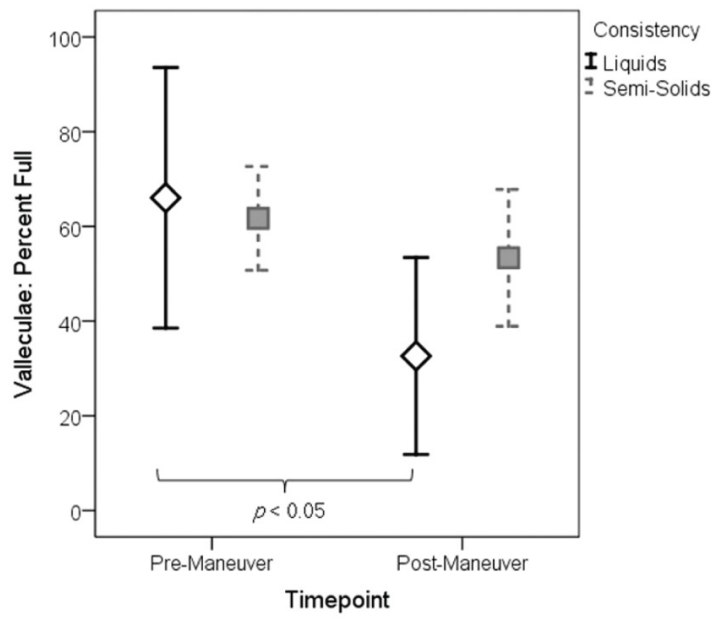

Figure 2. Pre- versus post-maneuver data for \%-full measures of residue in the valleculae

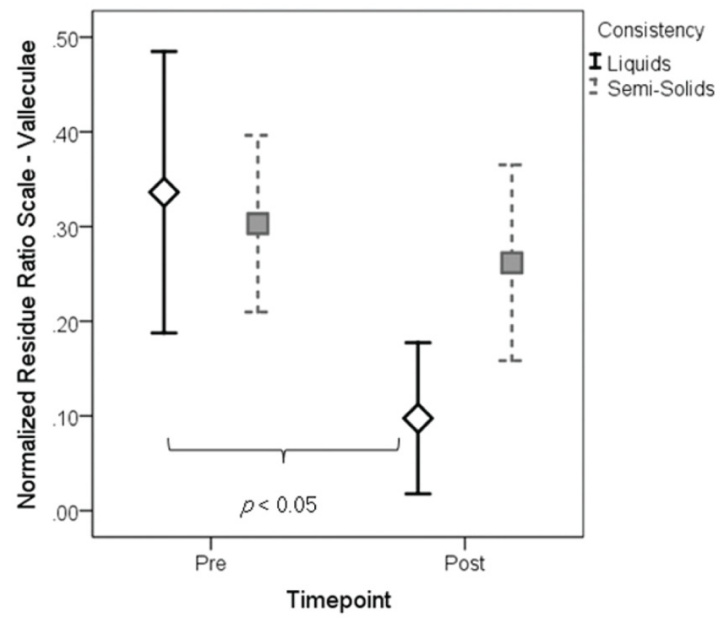

Figure 3. Pre- versus post-maneuver data for Normalized Residue Ratio Scale measures of residue in the valleculae

maneuver was successful in reducing residue. Furthermore, this reduction achieved clinical significance, lowering vallecular residue below a threshold previously reported to represent a risk for penetration-aspiration (i.e., NRSSv < 0.09) 11-29\% of the time, depending on bolus consistency. The maneuver showed greater effectiveness with thin and nectar-thick stimuli than with semi-solid stimuli. We speculate that this reflects the fact that the thin and nectar-thick stimuli flow more easily out of the valleculae when subjected to the squeezing effect of the maneuver.

\section{Limitations}

This study involves a fairly small sample size, arising from the strict inclusion criteria of only studying qualifying swallows, namely those where vallecular residue was present after an initial head neutral clearance swallow. The further exclusions of swallows involving the addition of new bolus material from the mouth (piecemeal deglutition) or pyriform sinus residue contributed to the small dataset of 19 qualifying liquid swallows and 46 qualifying semi-solid swallows available for analysis. Additionally, given the relatively small number of qualifying swallows, and the fact that qualifying residue was not observed consistently across all swallows of a given texture within an individual meant that it was not possible to explore the effect of the direction of the head turn, or to compare the effectiveness of this maneuver to any other maneuvers. Finally, given that an initial head-neutral clearing swallow was included prior to exploration of the head-turn-plus-chin-down technique, the data are limited to situations where pre-maneuver residue in the valleculae had an upper confidence interval limit less than $100 \%$ full.

\section{CONCLUSION}

The findings in this study show that the novel head-turn-pluschin-down maneuver can be an effective strategy for reducing persistent vallecular residue, particularly with thin and nectarthick liquids. The impact of reducing residue levels below a threshold that delineates aspiration risk holds particular clinical significance, but was only achieved in up to $29 \%$ of cases in this study. These preliminary data provide proof of concept, which should support the inclusion of the head-turn-plus-chindown maneuver as a compensatory technique to be explored in patients displaying vallecular residue in videofluoroscopy. Future research, perhaps incorporating manometric measurements, will help to elucidate the mechanisms behind the effectiveness of this maneuver. 


\section{ACKNOWLEDGEMENTS}

Support for this study was provided by Toronto Rehabilitation Institute - University Health Network, which receives funding under the Provincial Rehabilitation Research Program from the Ministry of Health and Long-term Care in Ontario. The views expressed do not necessarily reflect those of the Ministry.

\section{REFERENCES}

1. Perlman AL, Booth BM, Grayhack JP. Videofluoroscopic predictors of aspiration in patients with oropharyngeal dysphagia. Dysphagia. 1994;9(2):90-5. http://dx.doi.org/10.1007/BF00714593. PMid:8005013.

2. Palmer JB, Kuhlemeier KV, Tippett DC, Lynch C. A protocol for the videofluorographic swallowing study. Dysphagia. 1993;8(3):209-14. http:// dx.doi.org/10.1007/BF01354540. PMid:8359040.

3. Martin-Harris B, Brodsky MB, Michel Y, Castell DO, Schleicher M, Sandidge J, et al. MBS measurement tool for swallow impairment-MBSImp: establishing a standard. Dysphagia. 2008;23(4):392-405. http://dx.doi. org/10.1007/s00455-008-9185-9. PMid:18855050.

4. Rasley A, Logemann JA, Kahrilas PJ, Rademaker AW, Pauloski BR, Dodds WJ. Prevention of barium aspiration during videofluoroscopic swallowing studies: Value of change in posture. AJR Am J Roentgenol. 1993;160(5):10059. http://dx.doi.org/10.2214/ajr.160.5.8470567. PMid:8470567.

5. Shanahan TK, Logemann JA, Rademaker AW, Pauloski BR, Kahrilas PJ. Chin-down posture effect on aspiration in dysphagic patients. Arch Phys Med Rehabil. 1993;74(7):736-9. http://dx.doi.org/10.1016/00039993(93)90035-9. PMid:8328896.

6. Macrae P, Anderson C, Humbert I. Mechanisms of airway protection during chin-down swallowing. J Speech Lang Hear Res. 2014;57(4):1251-8. http:// dx.doi.org/10.1044/2014_JSLHR-S-13-0188. PMid:24686521.

7. McCulloch TM, Hoffman MR, Ciucci MR. High-resolution manometry of pharyngeal swallow pressure events associated with head turn and chin tuck. Ann Otol Rhinol Laryngol. 2010;119(6):369-76. PMid:20583734.

8. Ohmae Y, Ogura M, Kitahara S, Karaho T, Inouye T. Effects of head rotation on pharyngeal function during normal swallow. Ann Otol Rhinol Laryngol. 1998;107(4):344-8. http://dx.doi.org/10.1177/000348949810700414. PMid:9557771.

9. Balou M, McCullough GH, Aduli F, Brown D, Stack BC Jr, Snoddy $\mathrm{P}$, et al. Manometric measures of head rotation and chin tuck in healthy participants. Dysphagia. 2014;29(1):25-32. http://dx.doi.org/10.1007/ s00455-013-9472-y. PMid:23846323.

10. Bulow M, Olsson R, Ekberg O. Videomanometric analysis of supraglottic swallow, effortful swallow, and chin tuck in healthy volunteers. Dysphagia. 1999;14(2):67-72. http://dx.doi.org/10.1007/PL00009589. PMid:10028035.

11. Bulow M, Olsson R, Ekberg O. Videomanometric analysis of supraglottic swallow, effortful swallow, and chin tuck in patients with pharyngeal dysfunction. Dysphagia. 2001;16(3):190-5. http://dx.doi.org/10.1007/ s00455-001-0065-9. PMid:11453566.
12. Ertekin C, Keskin A, Kiylioglu N, Kirazli Y, On AY, Tarlaci S, et al. The effect of head and neck positions on oropharyngeal swallowing: a clinical and electrophysiologic study. Arch Phys Med Rehabil. 2001;82(9):1255-60. http://dx.doi.org/10.1053/apmr.2001.25156. PMid:11552200.

13. Castell JA, Castell DO, Schultz AR, Georgeson S. Effect of head position on the dynamics of the upper esophageal sphincter and pharynx. Dysphagia. 1993;8(1):1-6. http://dx.doi.org/10.1007/BF01351470. PMid:8436016.

14. Perlman AL, Grayhack JP, Booth BM. The relationship of vallecular residue to oral involvement, reduced hyoid elevation, and epiglottic function. J Speech Hear Res. 1992;35(4):734-41. http://dx.doi.org/10.1044/jshr.3504.734. PMid:1405528.

15. Park JM, Yong SY, Kim JH, Jung HS, Chang SJ, Kim KY, et al. Cutoff value of pharyngeal residue in prognosis prediction after neuromuscular electrical stimulation therapy for Dysphagia in subacute stroke patients. Ann Rehabil Med. 2014;38(5):612-9. http://dx.doi.org/10.5535/arm.2014.38.5.612. PMid:25379490.

16. Molfenter SM, Steele CM. The Relationship Between Residue and Aspiration on the Subsequent Swallow: An Application of the Normalized Residue Ratio Scale. Dysphagia. 2013;28(4):494-500. http://dx.doi.org/10.1007/ s00455-013-9459-8. PMid:23460344.

17. Pearson WG Jr, Molfenter SM, Smith ZM, Steele CM. Image-based measurement of post-swallow residue: the normalized residue ratio scale. Dysphagia. 2012;28(2):167-77. http://dx.doi.org/10.1007/s00455-0129426-9. PMid:23089830.

18. Ashford J, McCabe D, Wheeler-Hegland K, Frymark T, Mullen R, Musson $\mathrm{N}$, et al. Evidence-based systematic review: Oropharyngeal dysphagia behavioral treatments. Part III--impact of dysphagia treatments on populations with neurological disorders. J Rehabil Res Dev. 2009;46(2):195-204. http:// dx.doi.org/10.1682/JRRD.2008.08.0091. PMid:19533533.

19. McCabe D, Ashford J, Wheeler-Hegland K, Frymark T, Mullen R, Musson $\mathrm{N}$, et al. Evidence-based systematic review: Oropharyngeal dysphagia behavioral treatments. Part IV--impact of dysphagia treatment on individuals postcancer treatments. J Rehabil Res Dev. 2009;46(2):205-14. http://dx.doi. org/10.1682/JRRD.2008.08.0092. PMid:19533534

20. Wheeler-Hegland K, Ashford J, Frymark T, McCabe D, Mullen R, Musson $\mathrm{N}$, et al. Evidence-based systematic review: Oropharyngeal dysphagia behavioral treatments. Part II--impact of dysphagia treatment on normal swallow function. J Rehabil Res Dev. 2009;46(2):185-94. http://dx.doi. org/10.1682/JRRD.2008.08.0094. PMid:19533532.

21. Logemann JA. Manual for the videofluorographic study of swallowing. Austin, TX: Pro-Ed; 1986.

\section{Author contributions}

AN designed the study and led data collection, analysis and manuscript preparation. MPP assisted with data processing and analysis. TJV and AMN assisted with data analysis. CMS supervised the project and participated in manuscript preparation. 